\title{
Downlink Capacity of OFDMA-CR Based 5G Femtocell Networks
}

\author{
Joydev Ghosh*, Dushantha Nalin K. Jayakody*, Marwa Qaraqe ${ }^{\dagger}$ \\ ${ }^{*}$ School of Computer Science and Robotics, National Research Tomsk Polytechnic University, RUSSIA \\ ${ }^{\dagger}$ ICT Division, College of Science and Engineering, Hamad Bin Khalifa University, Qatar Foundation, QATAR \\ Email: joydev.ghosh.ece@gmail.com, nalin.jayakody@ieee.org, mqaraqe@hbku.edu.qa
}

\begin{abstract}
This research work explores small cell densification as a key technique for next generation wireless network (NGWN). Small cell densification comprises space (i.e, dense deployment of femtocells) and spectrum (i.e., utilization of frequency band at large). The usage of femtocells not only improves the spectral efficiency (SE) of the Heterogeneous two-tier networks against conventional approach, but also it alleviates outage probability and enhances the achievable capacity. We yield an analytical framework to establish the density of the femto base station (FBS) to a monotonically increasing or decreasing function of distance or radius, respectively. This ensures the enhanced performance in spectrum sharing Orthogonal Frequency Division Multiple Access (OFDMA) femtocell network models. We also illustrate the influence of active Femto users (i.e., users in femtocells, and they are usually low mobility and located closer to the cell centre with less fading), cluster size (i.e., a group of adjacent macrocells which use all of the systems frequency assignments) via simulation results.
\end{abstract}

Index Terms-Macrocell; Femtocell; Orthogonal frequency Division Multiple Access (OFDMA); Cognitive radio (CR) Technology; Downlink (DL) Capacity.

\section{INTRODUCTION}

The fourth generation $(4 \mathrm{G})$ wireless networks that are being deployed worldwide cannot fulfill the increasing requirement of smart technology facilitated device, i.e., smart watch, tablets and n-computing devices, in the next era [1], [2]. The 5G wireless networks enhance the area spectral efficiency (ASE), energy efficiency (EE), data rate (to almost around 5-8 Gbps), and supply a high quality of service (QoS) experience irrespective of the location of the device or which type of device is being used. Heterogeneous networks (HetNets) architecture contains a mixed use of infrastructure elements such as macrocells, femtocells, picocells, and relays to exchange data among the various types of cellular wireless and wireless local area network (WLAN) standards. The battery draining issue of smart phones, tablets can be reduced by means of energy efficient HetNets that will result in the elongation of battery life [3]. The concept of small cell wireless networks (femtocells, picocells, and macrocells), is attractive to mobile operators due to their ability to provide better indoor or outdoor local area system capacity and network coverage [4]. The base station (BS) of a femtocell uses a small transmit power to provide a network coverage of a distance of few meters. Femtocells are normally deployed at home or in a small commercial complex, and connected to cellular core networks over a digital subscriber line (DSL). The deployment of femtocells follows the activity and nature of the user, and traffic load distribution in the local area. Femtocells are dynamic and unplanned compare to other types of small cells that are deployed and fully controlled by operators. As observed from the research of cellular communications, rapid growth of capacity in wireless system can be attributed to a few main factors such as the number of wireless nodes, reuse of the radio spectrum, and enhancement in link efficiency. As of today, these three constituents continue to be the most influential parameters of rapid capacity growth in wireless networks. Multi-carrier networks can furthermore assist in optimizing the use of the spectrum as they permit putting the carriers in a non-contiguous manner. An abstract of several multi-carrier modulation techniques with regard to the optimum utilization in cognitive radio (CR) networks is described in [5]. The salient properties and the potential of the OFDMA technology makes it perfectly suitable for the CR based communication network. The prime concept of Orthogonal Frequency Division Multiple Access (OFDMA) scheme is that the accessible spectrum splits into many orthogonal sub-channels and allows more than one user to transmit data concurrently on the distinct sub-channels. However, the Doppler frequency offset in high mobility scenarios, where the pilot channel fluctuates at the time of communication, destroys the orthogonality of subcarriers [6]. This contributes to OFDMA sensitivity to inter carrier interference (ICI). The incorporation of CR technology and OFDMA is one of the best properties in next generation networks because of its adaptability in assigning resource among CR users [7]. The slow fading environment has been studied in [8] to analyse the SUs interference for CR technology.

In this paper, the femtocells with its less network coverage limitation having potential to provide large amount of simultaneous transmissions that can be satisfied in the system model, which revamps spatial reuse, thus potentially producing large network capacity. A femto base station (FBS) is a low power, small cellular base station, which can operate in both licensed and unlicensed band and it provides network service from 10 meters to 100 meters [17]. The user traffic is distributed among the femtocells by changing their coverage area and cluster size depending on the difference between each cell load. Power and spectrum resources have been allocated under power constraint to maximize the capacity of the femtocell users in DL transmission, and present the simulation outcomes 
to evaluate the performance. Here, we articulate more on the analytical results of the DL capacity for dual-layer femtocell networks under different metrics and hence it is distinct from the quite similar types of works concentrated on uplink (UL) capacity [18] or cellular ad hoc network [19], [20]. By applying Poission point process (PPP) model, limits on the distribution of estimated average interference from dual-layer spatial point processes are successfully computed. The large overall capacity in association with the large femto-BS density fulfilling a per-layer outage limit can be determined. The major contributions of the paper can be summarized as:

1) It is shown that the capacity set by the interfering transmitter is consistent with the signal to interference plus noise ratio, $(\mathrm{S} /(\mathrm{I}+\mathrm{N}))$ at the sufferer receiver. This approach needs information of several transmission factors of the interfering signal, such as the degree of modulation, OFDMA, sub-carrier spacing etc.

2) We incorporate the idea of $\mathrm{CR}$ into spectrum sharing two tier OFDMA networks where active femtocells are responsible for increasing the overall capacity of the network. Femtocells in the adjacent apartments use orthogonal one third spectrum in the cell edge area, where $\mathrm{FRF}=3$ [9], configured via network planning. The capacity of Femto users depends on the number of accessible channels, and the number of Femto users that have data to transmit, which are defined as active Femto users in this paper.

This paper is organized as follows: The system model is defined in Section II. Section III discusses the simulation model. Finally, results and discussions are provided in Section IV before concluding remarks in Section V.

\section{SySTEM MODEL}

Table I: Table of Notations

\begin{tabular}{|l|l|}
\hline Symbol & Meaning \\
\hline$F$ & The set of FBS \\
\hline$M_{i}$ & The set femto users (FUs) in FBS $i$ \\
\hline$N_{i}$ & The set of available channels in FBS i \\
\hline$i$ & The index of FBS \\
\hline$j$ & The index of FU \\
\hline$c$ & The index of channel \\
\hline$m_{i}$ & $\begin{array}{l}\text { The number of FU in FBS } i \\
\text { in FBS } i\end{array}$ \\
\hline$\alpha_{i j c}$ & $\begin{array}{l}\text { The transmission power for FBS } i \text { at } \\
\text { channel } c \text { on FU } j\end{array}$ \\
\hline$p_{i j c}$ & $\begin{array}{l}\text { The channel gain on channel } c \text { for FBS } i \\
\text { and FU } j\end{array}$ \\
\hline$h_{i j c}$ & The minimum required SINR for FUs \\
\hline$\psi$ & Two dimensional Euclidean plane \\
\hline$|\mathbf{R}|^{2}$ & SINR threshold of a MU \\
\hline$\eta$ & The maximum allowed femto-BS density \\
\hline$\hat{\lambda}_{f}$ & $\begin{array}{l}\text { Large Scale Path Loss (LSPL) between a } \\
\text { referenced MU and its serving MBS }\end{array}$ \\
\hline$R_{M}^{-\alpha}$ &
\end{tabular}

In this section, we initiate the system model and assumptions for next generation dual layer HetNets, and formulate the DL spectrum sharing issue. The notations considered in this paper are shown in Table I. Suppose a set of $F$ FBSs are present in the network coverage of macro base station (MBS). For any FBS $i(i \in F)$, there are a set of $M_{i}$ FUs. Ordinarily, the numbers of FUs are in between two and four, as indicated in [10]. According to the homogeneous spatial PPP model the small cells (and the mobile users) are uniformly scattered in two-dimensional Euclidean plane denoted $|\mathbf{R}|^{2}$. The actual number of small cells follows a Poisson distribution with density $\lambda$. The mean value of the small cells is given by $N_{s}=\lambda|\mathbf{R}|^{2}$. In this model, we assume that the process is homogeneous, thus the points are scattered uniformly in the plane. In this case, the intensity parameter $\lambda$ is constant in every part of $|\mathbf{R}|^{2}$ [11]. Any femtocell user receives interference from neighbouring femtocells, which are using the same channel.

\section{A. Interference Analysis of an Macro user (MU)}

As there is no interference within the cell, we let consider that the received interference for the reference macro MU utilizing sub-channel $n$ is from adjacent MBSs and nearby FBSs. Here, nearby interfering FBS refers to the FBS whose position is less than a certain distance between MUs. Moreover, crosstier interference generated from other FBSs is neglected for long distance.

In the signal to interference plus noise ratio (SINR), numerator part consists of the received signal strength and denominator part is the summation of three components (i.e., summation of interference generated due to inter-tier and intratier interference, and static thermal noise).

Therefore, the SINR of MU $i$ located at the edge of the serving area (i.e., $R_{M}$ ) using sub-channel $n$ can be expressed as

$$
\operatorname{SINR}_{M_{i}}^{n}=\frac{p_{M_{i}}^{n}\left|h_{M_{i}}^{n}\right|^{2} R_{M}^{-\alpha}}{\rho_{F_{j}}^{n} p_{F_{i}}^{n}\left|h_{F_{j}, M_{i}}^{n}\right|^{2} d_{F_{j}, M_{j}}^{-\alpha}}+,
$$

where $\rho_{F_{j}}^{n}=1$ and $\rho_{M_{k}}^{n}=1$ denote that there is co-channel interference from $\operatorname{FBS} j$ and MBS $k$ to the MU $i$ on the sub-channel $n$ respectively, else, it is $0 ; \alpha$ indicates pathloss exponent. Here, $\left|h_{M_{i}}^{n}\right|^{2}$ denotes the generated channel gain between $i^{\text {th }}$ MBS and respective $i^{\text {th }}$ MU over the $n^{\text {th }}$ subchannel, whereas $\left|h_{F_{j}, M_{i}}^{n}\right|^{2}$ denotes the generated channel gain between $j^{\text {th }}$ FBS and $i^{\text {th }}$ MBS to the $i^{\text {th }}$ MU, and $\left|h_{M_{k}, M_{i}}^{n}\right|^{2}$ denotes generated channel gain between $j^{\text {th }} \mathrm{MBS}$ and $i^{\text {th }}$ MBS to the $i^{\text {th }} \mathrm{MU} ; p_{M_{i}}^{n}, p_{F_{i}}^{n}, p_{M_{k}}^{n}$ denote transmit power by the corresponding base station entity that facilitates service over the $n^{\text {th }}$ sub-channel respectively, $d_{F_{j}, M_{j}}^{-\alpha}$ is the distance between a MU and its associated FBS, $d_{M_{k}, M_{i}}^{-\alpha}$ is the distance between a $\mathrm{MU}$ and its adjacent $\mathrm{MBS}, N_{0}$ stands for noise power.

\section{B. Interference Analysis of an Femto User (FU)}

The received interference of a FU is large as adjacent MBSs in contrast to the generated interference from the FBSs 


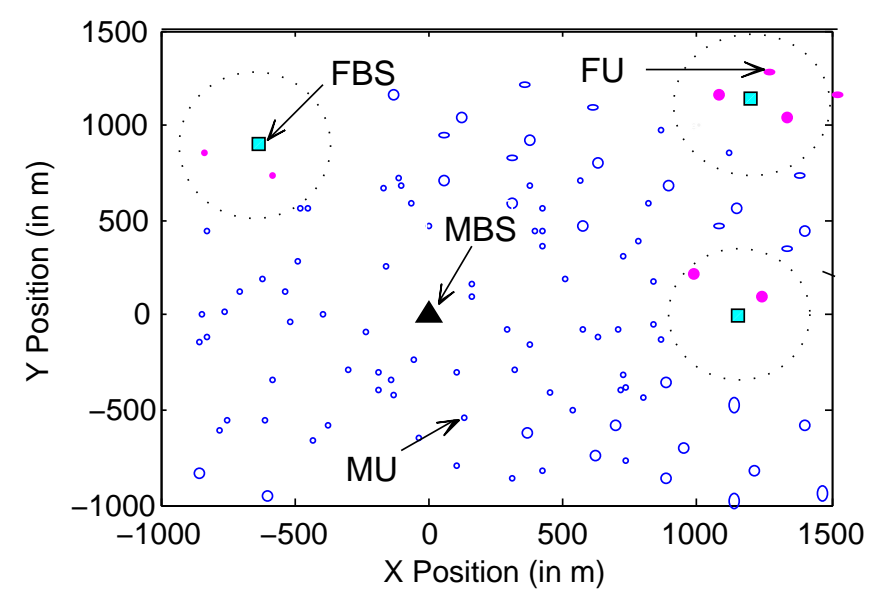

Fig. 1: Co-existence scenario of cognitive femtocell networks under macrocell infrastructure

located at the same macrocell or at the adjacent macrocells, respectively. Hence, we neglected the interference introduced due to the FBSs located at the same macrocell or at the adjacent macrocells, respectively, as it has a minimum effect on the total interference. Thus, SINR of FU $i^{\text {th }}$ can be written as:

$$
\begin{aligned}
& \operatorname{SIN} R_{F_{i}}^{n}= \frac{p_{F_{i}}^{n}\left|h_{F_{i}, F_{i}}^{n}\right|^{2} R_{f}^{-\alpha}}{\sum_{j=1}^{N_{c}} \rho_{M_{j}}^{n} p_{M_{j}^{n}}\left|h_{M_{j}, F_{i}}^{n}\right|^{2} d_{M_{j} F_{i}}^{-\alpha}} \\
& \quad+\sum_{k=2}^{N_{c}} \rho_{F_{k}}^{n} p_{F_{k}}^{n}\left|h_{F_{k}, F_{i}}^{n}\right|^{2} d_{K_{j} F_{i}}^{-\alpha}+N_{0}
\end{aligned}
$$

Suppose a set of $F$ FBSs are there in the network coverage of MBS. For any FBS $i(\forall i \in F)$, there are a set of $M_{i}$ FUs. In this paper, we use notation $i$ to denote the femtocell for the purpose to track the location of FBS. We make use of a set of $N_{i}$ licensed channels that have been made available for femtocell $i$.

The DL capacity of $j^{\text {th }} \mathrm{FU}$ in an $i^{\text {th }}$ femtocell can be given by Shannon's capacity formula as follows:

$$
C_{i j}=\sum_{c \in N_{i}} B \log _{2}\left(1+\beta_{i j c} S I N R_{F_{i}}^{n}\right)
$$

where $\beta_{i j c}$ is a binary indicator. If $\beta_{i j c}=1$, user $j$ in femtocell $i$ works on channel $c$, zero otherwise.

Therefore, we can express the DL capacity of a $j^{\text {th }}$ femtocell that is the sum total of all the capacity of its users

$$
C_{i}=\sum_{j \in M_{i}} \sum_{c \in N_{i}} B \log _{2}\left(1+\beta_{i j c} S I N R_{F_{i}}^{n}\right), \forall i \in F .
$$

\section{DL Spectrum sharing}

In this paper, we contemplate the DL spectrum sharing issue, where FBSs use the licensed channels keeping in mind that the licensed channels are not being used by macrocell user. Hence, there is no inter-channel interference between macrocells and femtocells. The only interference can be minimized at large is the co-tier interference among the FBSs. Let us consider that each FU in a femtocell needs one channel. We articulate the worst case where all nearby FBSs are in DL transmission. Here, we analyze the DL capacity and then prepare methodically the spectrum sharing issue. The spectrum sharing issue in cognitive femtocell DL transmission is to optimize the DL capacity of all FBSs keeping the parameters such as channel allocation [44], SINR, and power constraints on the desired value.

$$
P: \max \sum_{j \in F} C_{i}
$$

Subject to: $\beta_{i j c} \in\{0,1\}, \quad \forall j \in F, i \in N_{f u}, c \in N_{S C}$

$$
\begin{aligned}
& \sum_{c \in N_{S C}} \beta_{i j c}=1, \quad \forall j \in F, i \in N_{f u} \\
& \sum_{i \in N_{f u}} \sum_{c \in N_{S C}} \beta_{i j c}=1, \quad \forall i \in F \\
& \beta_{i j c} \geq \psi, \text { if } \beta_{i j c}=1, \quad \forall j \in F, i \in N_{f u}, c \in N_{S C} \\
& p_{i j c}=0, \text { if } \beta_{i j c}=0, \quad \forall j \in F, i \in N_{f u}, c \in N_{S C} \\
& p_{i j c}>0, \quad \forall j \in F, i \in N_{f u}, c \in N_{S C} \\
& \sum_{i \in N_{f u}} \sum_{c \in N_{S C}} p_{i j c} \leq P_{i}^{\text {max }}, \quad \forall j \in F
\end{aligned}
$$

where $\psi$ indicates the minimum desired SINR for FUs and $p_{i j c}$ indicates power transmitted by femtocell $j$ for FU $i$ on channel $c$. A limitation in (6) signifies every user in this cognitive femtocell scenario can only use one channel. A limitation in (7) signifies the maximum number of channels can be used in a femtocell is equal to the number of users in that femtocell. A limitation in (8) indicates that if channel $c$ is assigned to user $j$ in femtocell $i$ for DL transmission, the SINR achieved on user $j$ should be larger than the threshold, $\psi$ established in advance. A limitation in (9) means any FBS $i$ shall not assign any power on channel $c$, if channel $c$ is not assigned to FBS $i$. A limitation in (10) indicates the transmission power of an FBS should not be less than 0 , whereas a limitation in (11) denotes the total transmission power of an FBS $i$ on its FUs can not exceed the maximum power transmission $P_{i}^{\max }$.

Theorem 1 The active FBS density should be smaller than $\hat{\lambda}_{f}=\frac{\left[-\ln \left[(1-\epsilon)\left(1+\frac{\eta \rho_{F_{j}}^{n} d_{f}^{-\alpha}}{R_{m}^{-n}}\right)\right]-\frac{\eta N_{0}}{p_{M} R_{m}^{-n}}\right]}{R_{m}^{2} \eta^{\delta} \rho_{F_{j}}^{n} K_{\beta}}$ to neglect interference from FBSs violating the outage constraint at a MU in the macrocell with per tier outage probability $\epsilon$ and area radius of serving networks, $R_{m}$.

Proof:Taking into account that the MU located at the macro- 


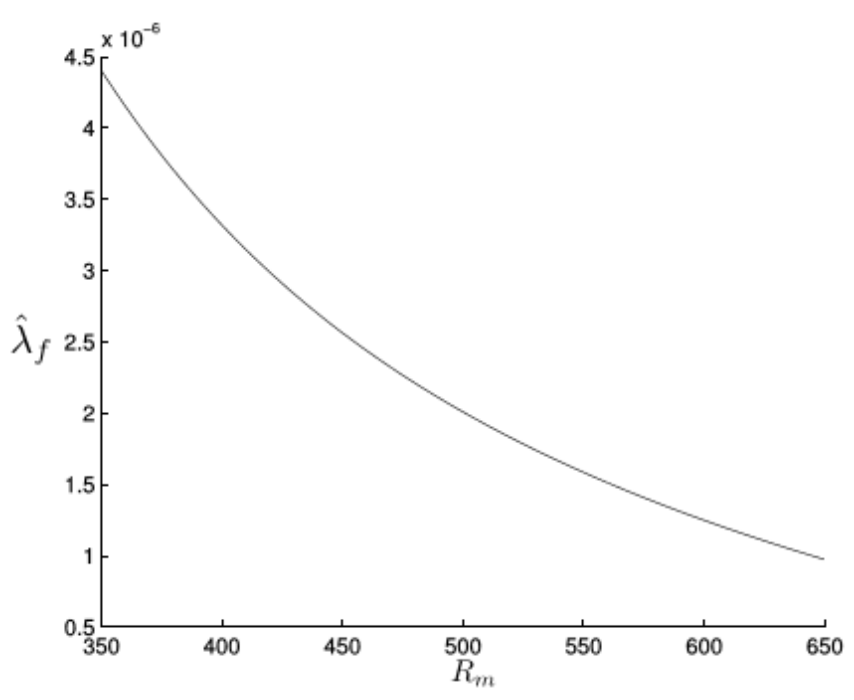

Fig. 2: The effect of $R_{m}$ on $\hat{\lambda}_{f}$.

cell edge, the following outage restriction should be followed:

$$
\begin{aligned}
& \mathbf{P}\left(\begin{array}{c}
\frac{p_{M_{i}}^{n}\left|h_{M_{i}, M_{i}}^{n}\right|^{2} R_{m}^{-\alpha}}{\rho_{F_{j}}^{n} p_{F_{i}}^{n}\left|h_{F_{j}, M_{i}}^{n}\right|^{2} d_{F_{j}, M_{j}}^{-\alpha}} \\
+\sum_{k=2}^{N_{c}} \rho_{M_{k}}^{n} p_{M_{k}}^{n}\left|h_{M_{k}, M_{i}}^{n}\right|^{2} d_{M_{k}, M_{i}}^{-\alpha}+N_{0}
\end{array}\right) \eta \eta \\
& =1-\epsilon .
\end{aligned}
$$

Moment Generating Function (MGF) of interference can be expressed from [13] as:

$$
\begin{aligned}
\mathbf{E}[\exp (-\eta I)] & =\exp \left(-2 \pi \hat{\lambda}_{f} \int_{0}^{\infty} \frac{x}{1+\frac{x_{\alpha}}{\eta p_{M_{i}}^{n}}}\right) \\
& =\exp \left(-\hat{\lambda}_{f} p_{M_{i}}^{n} \eta^{\frac{2}{\alpha}} K_{\beta}\right),
\end{aligned}
$$

where, $\left.K_{\beta}=\frac{2 \pi^{2}}{\alpha \sin \left(\frac{2 \pi}{\alpha}\right)}\right)$.

The left hand side of (11) can be assessed by applying (12) as

$$
\begin{gathered}
\mathbf{P}\left(\left|h_{M_{i}, M_{i}}^{n}\right|^{2} \geq \frac{\eta}{p_{M_{i}}^{n} R_{m}^{-\alpha}}\left(\rho_{F_{j}}^{n} p_{M_{i}}^{n}\left|h_{F_{j}, M_{i}}^{n}\right|^{2} d_{F_{j} M_{i}}^{-\alpha}\right.\right. \\
\left.\left.+\sum_{k=2}^{N_{c}} \rho_{M_{k}}^{n} p_{M_{k}}^{n}\left|h_{M_{k}, M_{i}}^{n}\right|^{2} d_{M_{k}, M_{i}}^{-\alpha}+N_{0}\right)\right) \\
=\exp \left(-\frac{\eta N_{0}}{p_{F_{i}}^{n} R_{m}^{-\alpha}}\right) \exp \left(-\hat{\lambda}_{f} R_{m}^{2}\left(\frac{\eta p_{M_{j}}^{n} \rho_{M_{j}}^{n}}{p_{F_{i}}^{n}}\right)^{\delta} K_{\beta}\right) .
\end{gathered}
$$

Therefore, we get:

$$
\hat{\lambda}_{f}=\frac{\left[-\ln \left[(1-\epsilon)\left(1+\frac{\eta \rho_{F_{j}}^{n} d_{f}^{-\alpha}}{R_{m}^{-\alpha}}\right)\right]-\frac{\eta N_{0}}{p_{M} R_{m}^{-\alpha}}\right]}{R_{m}^{2}} \eta^{\delta} \rho_{F_{j}}^{n} K_{\beta} .
$$

Corollary1: The highest active FBS density $\hat{\lambda}_{f}$ is a monotonically decreasing function of $R_{m}$ when the necessity of providing QoS to all MUs is satisfied.

Fig. 2 depicts the effects of $R_{m}$ on $\hat{\lambda}_{f}$ and the corresponding $\hat{\lambda}_{f} \approx 3.8 \times 10^{-6}$ for $R_{m}=400$. The system parameters are set as $N_{0}=10^{12}, \alpha=4, \epsilon=0.1$, and $\rho_{F_{j}}^{n}=0.01$, they are applied in the entire simulation outcomes in the following figures. It can be noticed that $\mathrm{d}$ should be the initial retrieved to evaluate $\hat{\lambda}_{f}$ at each FBS in a distributed fashion. Now, $R_{m}$ can simply be computed either by exchanging its own $R_{m}$ with adjacent FBSs or with the MBS by recording $R_{m}$ for all FBSs and periodically transmitting the information to every FBS. Each FBS uses Theorem 1 to compute the highest active FBS density to make sure the QoS of both MUs and FUs.

Lemma 1: The active FBS density should be smaller than

$$
\hat{\lambda}_{f}=\frac{\left[-\ln (1-\epsilon)-\left(\frac{\eta N_{0}}{p_{M} R_{f}^{-\alpha}}\right)\right]}{R_{f}^{2}\left(\frac{\eta p_{M_{j}}^{n} \rho_{M_{j}}^{n}}{p_{F_{i}}^{n}}\right)^{\delta} K_{\beta}}
$$

to neglect interference from FBSs violating the outage constraint at a FU.

Proof: To guarantee the outage constraint, an FU positioned at its serving femtocell edge with the smallest distance to the MBS should be taken into account as it suffers from the serious cross-tier interference. The following outage constraint should be followed:

$$
\mathbf{P}\left(\frac{p_{F_{i}}^{n}\left|h_{F_{i}, F_{i}}^{n}\right|^{2} R_{f}^{-\alpha}}{I_{M}^{F}+N_{0}} \geq \eta\right)=1-\epsilon,
$$

where, $I_{M}^{F}=\sum_{j=1}^{N_{c}} \rho_{M_{j}}^{n} p_{M_{j}}^{n}\left|h_{M_{j}, F_{i}}^{n}\right|^{2} d_{M_{j}, F_{i}}^{-\alpha}+$ $\sum_{k=2}^{N_{c}} \rho_{F_{k}}^{n} p_{F_{k}}^{n}\left|h_{F_{k}, F_{i}}^{n}\right|^{2} d_{K_{j} F_{i}}^{-\alpha}+N_{0}$ is the generated interference from the surrounding MBS and adjacent FBS to the referenced FBS. The left hand side of (15) can be expressed as

$$
\begin{aligned}
& \mathbf{P}\left(\left|h_{F_{i}, F_{i}}^{n}\right|^{2} \geq \frac{\eta}{p_{F_{i}}^{n} R_{f}^{-\alpha}}\left(I_{M}^{F}+N_{0}\right)\right) \\
& =\exp \left(-\frac{\eta N_{0}}{p_{F_{i}}^{n} R_{f}^{-\alpha}}\right) \exp \left(\hat{\lambda}_{f} R_{f}^{2}\left(\frac{\eta p_{M_{j}}^{n} \rho_{M_{j}}^{n}}{p_{F_{i}}^{n}}\right)^{\delta} K_{\beta}\right) .
\end{aligned}
$$

We thus get,

$$
\hat{\lambda}_{f}=\frac{\left[-\ln (1-\epsilon)-\left(\frac{\eta N_{0}}{p_{M} R_{f}^{-\alpha}}\right)\right]}{R_{f}^{2}\left(\frac{\eta p_{M_{j}}^{n} \rho_{M_{j}}^{n}}{p_{F_{i}}^{n}}\right)^{\delta} K_{\beta}} .
$$

Corollary 2: The highest active FBS density is a monotonically decreasing function of $R_{f}$, when the necessity of providing QoS to all FUs is satisfied.

Corollary 2 can represent from the numerical outcomes illustrated in Fig. 3 where $P_{m}=25$. Here, $R_{f}$ is possible to find at FBS by the received power of the transmitted signal from the MBS and the corresponding $\hat{\lambda}_{f} \approx 0.35 \times 10^{4}$ for 


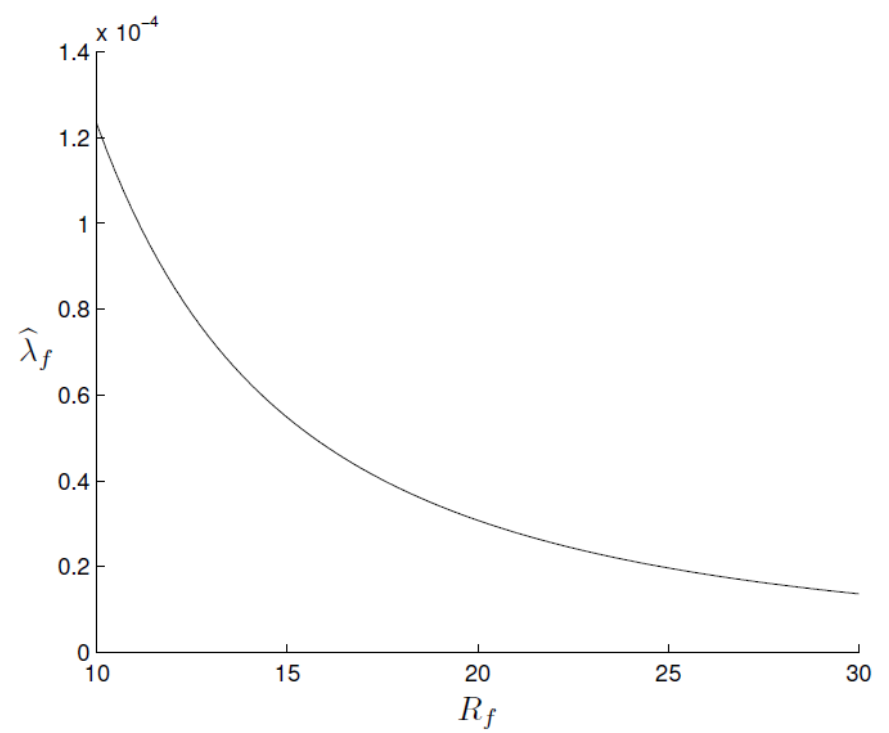

Fig. 3: The effect of $R_{f}$ on $\hat{\lambda}_{f}$.

Table II: 5G Physical Layer System Parameters

\begin{tabular}{|l|l|}
\hline Parameter & Value \\
\hline Total transmit power & $26 \mathrm{dBm}$ \\
\hline System bandwidth & $200 \mathrm{MHz}$ \\
\hline Carrier Frequency & $3.5 \mathrm{GHz}$ \\
\hline Sub-carrier spacing & $100 \mathrm{kHz}$ \\
\hline Eff. sub-carrier & 3200 \\
\hline Frame length & $250 \mathrm{ps}$ \\
\hline Symbol time & $16.67 \mathrm{ps}$ \\
\hline OFDM Symbols per frame & 50 \\
\hline Highest order modulation & $256 \mathrm{QAM}$ \\
\hline Stationary Thermal Noise $\left(N_{0}\right)$ & $-174 \mathrm{dBm}$ \\
\hline
\end{tabular}

$R_{f}=20$. A limitation for the active FBS density can be computed from the respective FBS. When an FU reuses the spectrum allocated for macrocell to receive data, the achieved SINR largely depends on the distance between itself and MBS, which is approximated by the distance between its serving FBS and the MBS. Therefore, the received SINR at the FBS is also limited by interference from neighbouring FBSs, and Lemma 1 provides a detail account of the permissible FBS density.

\section{Simulation Model}

(i) In this article, the upgraded dual layer network aids a higher MCS index 256QAM; thus, the maximum DL capacity for a $5 \mathrm{G}$ wireless network has been significantly improved. (ii) Here, we have applied OFDMA, which is a multi-user type of OFDM. Multiple access is possible in HetNets by allocating subsets of sub-carriers to each user equipments (UEs) for a fixed number of physical resource block (PRB), thus permitting simultaneous transmissions from multiple UEs. (iii) An equal power transmission to individual user has been considered; whereas the distribution of each UE transmission power on the sub-carriers is achieved by the power control scheme. The 10 UEs have been dropped at 300 different positions with 10 channel realizations per drop. The highest

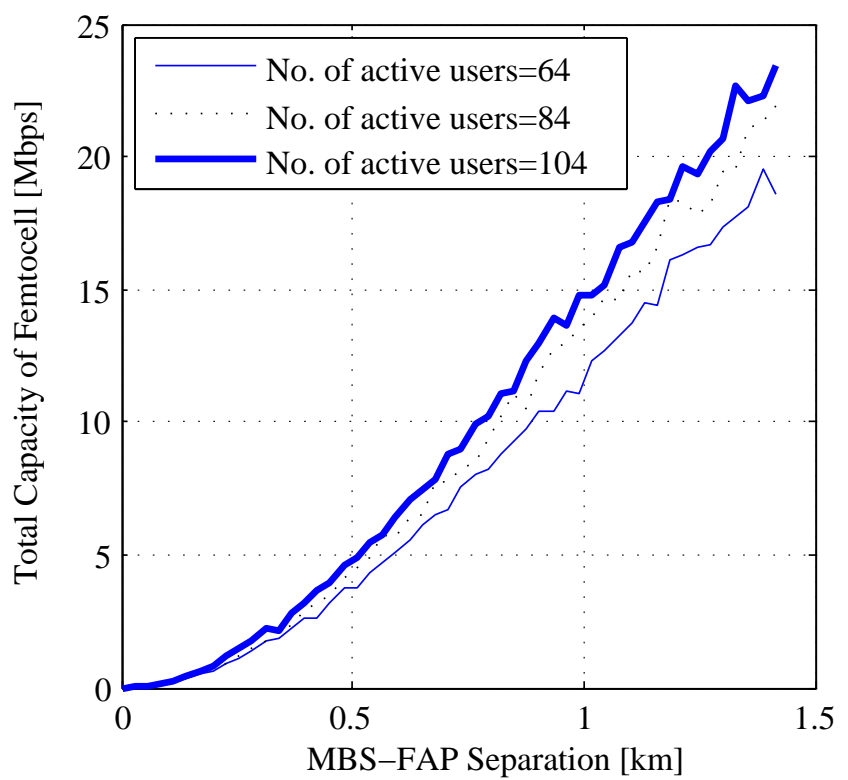

Fig. 4: Effects of active users on the transmission capacity of a femtocell

achievable spectral efficiency for 10 UEs based upon the physical layer parameters listed in Table-II is:

$$
10 \frac{\log _{2} 2563200}{250 p s 200 M \mathrm{~Hz}}=5.12 \mathrm{Mbps} / \mathrm{Hz}
$$

(iv) The simulation executed by running an iterative approach and each iteration has 25 sub-frames. In every iteration, the FBSs and FUs had been distributed independently. The number of users taking advantage of FBS and MBS considered to be 20 and 60, respectively. Other related simulation variables have been summarized in Table II.

(v) In each iteration, the channel allocation process shall upgrade according to the recently measured interference.

\section{Simulation Results and Discussion}

This section uses simulation outcomes to validate the accuracy of the proposed analytical models of OFDMA-CR based femtocell networks. Fig. 4 shows that the DL femtocell capacity for $5 \mathrm{G}$ wireless networks is influenced by the number of linked UE devices. If the number of active users grows from one to many, the DL capacity of a femtocell increase for $5 \mathrm{G}$ wireless networks. The femtocell capacity with 104 numbers of active UE devices at a location gap of $1 \mathrm{~km}$ between MBS and FAP is $15-17$ Mbps higher than that of 84 or 64 numbers of active UE devices in a femtocell. Fig. 5 depicts the effects of cluster size on the DL capacity of a femtocell, where the capacity does not deteriorate even though the cluster size becomes large. The average number of femtocell active users varies between 64 and 104, and femtocell deployment densities in the form of different cluster size are considered in this plot. In Fig. 6, the total capacity of femtocell of dualtier networks is shown against the separation between MBS and FAP in which different femtocell coverage radius is set 


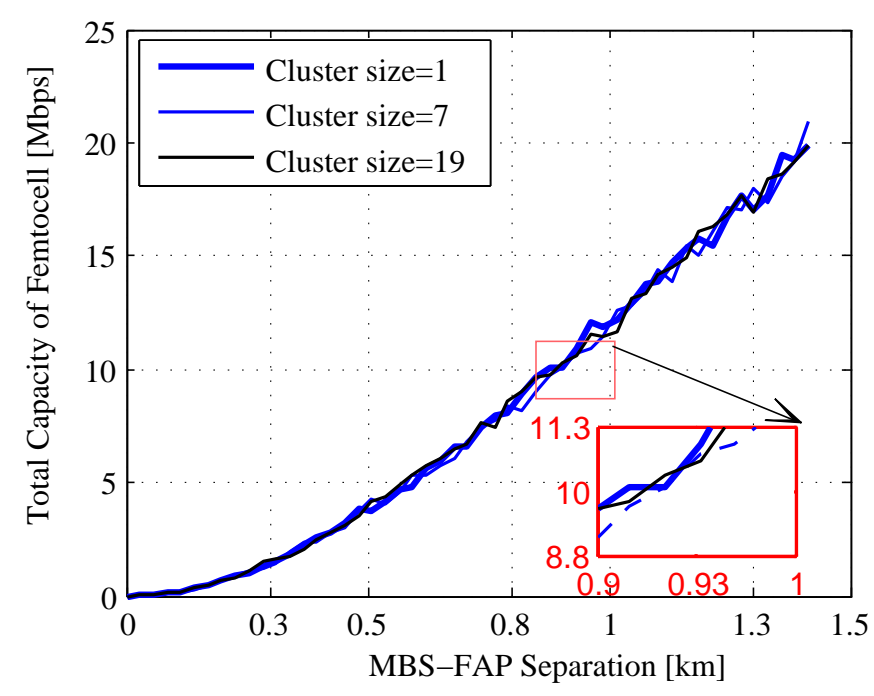

Fig. 5: Total capacity of femtocell achieves by incorporating the effect of cluster size into the device.

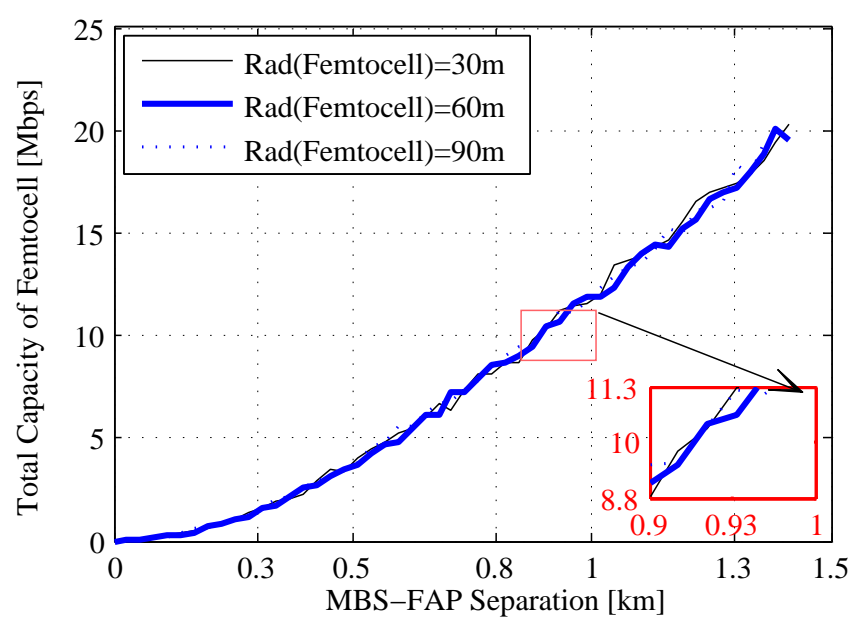

Fig. 6: Effects of femtocell radius on the transmission capacity of a femtocell.

of simulation. As $R_{m}$ increases, the reference active MUE positioned at the edge area of the macrocell receives a weaker signal from the MBS. To maintain the outage constraint at the reference active MUE for providing QoS, the density of active FBSs can be increased which agreed with the analytical views of Corollary1.

Fig.7 shows the performance of total capacity of femtocell as path loss exponent increases. The highest capacity is obtained under frequency reuse factor $(F R F)=3$ with number of femtocell being deployed, and capacity decreases as the path loss exponent increases for the increasing separation distance between MBS and FAP.

Interference measurements performed at dual layer HetNets under the presence of active users are shown in Fig. 8. The Active UEs measuring large interference are more likely to cause large interference, so DL transmit power is decreased

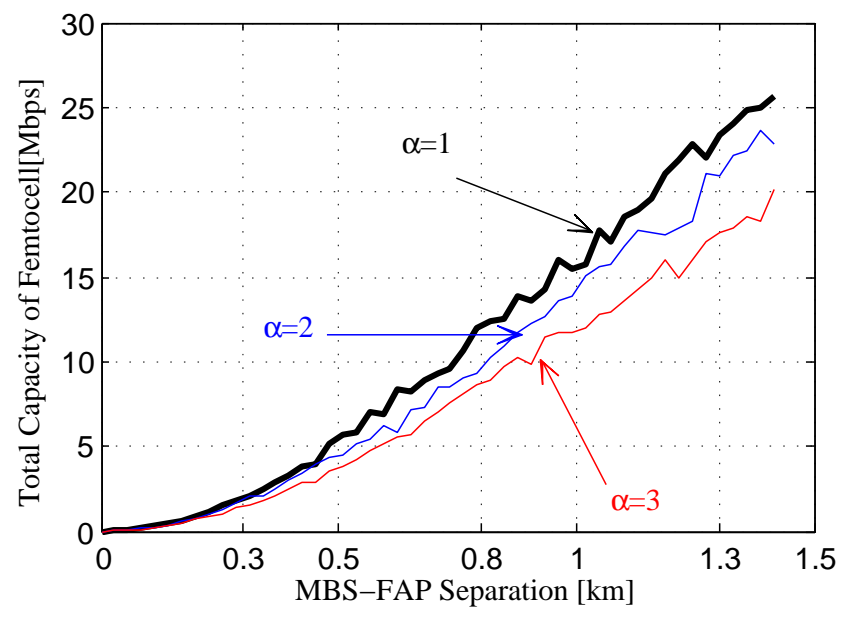

Fig. 7: Effects of pathloss exponent on the total capacity of a femtocell.

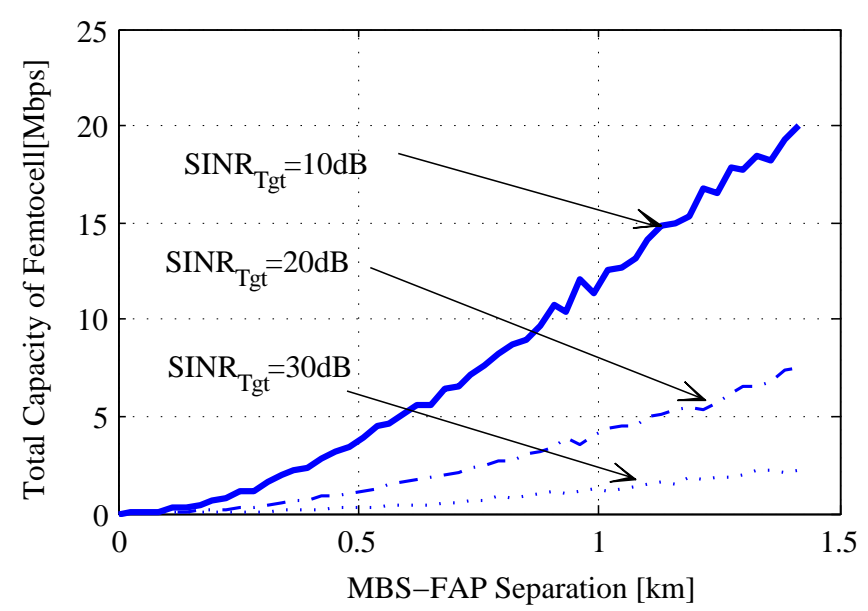

Fig. 8: Effects of target SINR on the transmission capacity of a femtocell.

depending upon the fractional compensation of the obtained interference. The DL power control of $5 \mathrm{G}$ networks indicates DL transmit powers so that number of floors in the path and interference are compensated and a set signal quality (i.e., a target SINR), is obtained at the UE receiver. This fractional compensation of the measured interference is in tune with the target SINR when large interference is observed. As such, femtocell capacity degrades according to the increase of target SINR.This is due to the fact that when target SINR becomes large, the density of active FBSs decreases to ensure the pertier outage constraint, resulting in less transmission capacity.

Fig.9 shows that the simulation results of the achievable capacity of femtocell closest match for the use of different number of sub-carriers. We find the presence of limited discrepancy mainly due to the cross-tier interference received by the FU from the MBS that is approximated by the receiver at the FBS. Here, femtocell users use shared sub-carriers to mitigate intra-tier interference and maximizing the capacity. 


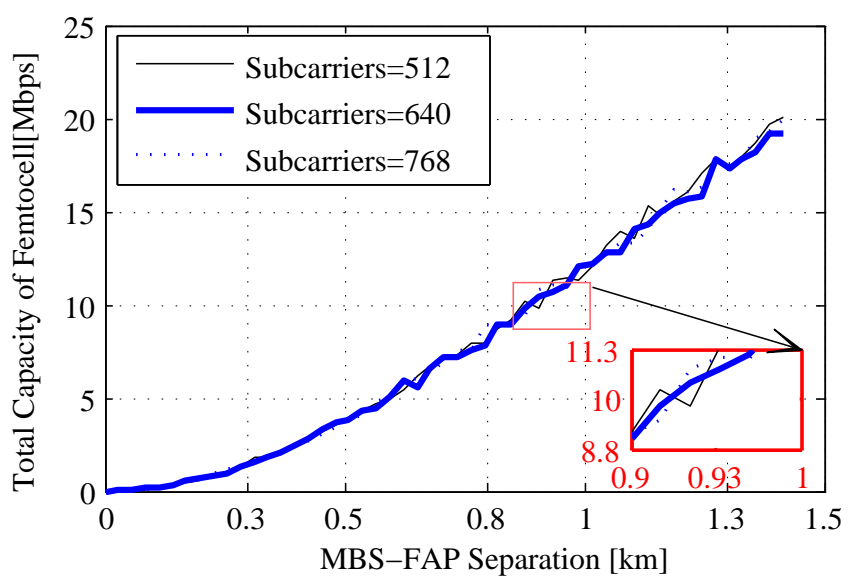

Fig. 9: Effects of sub-carriers on the transmission capacity of a femtocell.

\section{CONCLUSION}

Quite a few network parameters such as sub-carrier selection, active user, cluster size and power controls have been considered to deal with the challenging issues created from network densification. Here, dense HetNets have been established to substantially increase user capacity in dense networks with a large number of users and devices. With implementable property and intractable analytical outcomes, the proposed model facilitates the deployment and development of CR in OFDMA based femtocell networks under macrocell infrastructure.

\section{REFERENCES}

[1] Boccardi, F., Robert W. Heath Jr., Lozano, A., Marzetta, T. L., Popovski, P., "Five Disruptive Technology Directions for 5G," IEEE Communications Magazine, vol.52, no.2, pp.-74-80, Feb 2014.

[2] Agyapong, P. K.,Iwamura, M., Staehle, D., Kiess, W., and Benjebbour, A., "Design Consideration for a 5G Network Architecture," IEEE Communications Magazine, vol.52, no.11, pp.65-75, Nov.2014.

[3] Ghosh, J., Roy, S. D., "Qualitative analysis for coverage probability andenergy efficiency in cognitive-femtocell networks under macrocellinfrastructure,” Electronics Letters, vol.51, issue 17 @ IET 2015.
[4] Ghosh, J., Roy, S. D., "The Implications of Cognitive Femtocell Based Spectrum Allocation Over Macrocell Networks," Wireless Personal Communications(Springer US), DOI:10.1007/s11277-016-3597-x,13th Aug' 16.

[5] Kollar, Z. S., Horvath, P.,"Modulation schemes for cognitive radio in white spaces," Radioengineering, 2010, vol. 19, no.4, p.511-517.

[6] TAO, C., QIU, J., LIU, L.,"A novel channel estimation algorithm with ICI mitigation over fast fading channels," Radio Engineering, vol.19, no.2, June 2010, pp.347-355.

[7] Haykin, S., "Cognitive radio: brain empowered wireless communications," IEEE Journa of Selected Areas in Communication, 2005, vol.23, no.2, pp.201-220.

[8] Ruttik, K., Koufos, K., Jantti, R.,"Modeling of the secondary systems generated interference and studying of its impact on the secondary system design," Radio Engineering, 2010, vol.19, no.4, pp.488-493.

[9] Ghosh, J., Jayakody, D. N. K., Qaraqe, M., Tsiftsis, T. A.,"Coverage Probability Analytics by Fractional Frequency Reuse Scheme," 1st International Telecommunications conference ITELCON 2017 (Springer Lecture Notes in Electrical Engineering), Istanbul, Turkey, Dec 2017.

[10] Chandrasekhar, V., Andrews, V., and Gatherer, V. "Femtocell networks: A survey," IEEE Commun. Mag., vol. 46, no. 9, pp. 5967, Sep. 2008.

[11] Ghosh, J., Jayakody, D.N.K, and Qaraqe, M.,"Cognitive-Femtocell Based Resource Allocation in Macrocell Network," PIMRC 2017, Montreal, QC, Canada.

[12] Digital Mobile Radio Towards Future Generation Systems Cost 231 Final Report COST Action 231, 1999.

[13] Baccelli, F., Blaszczyszyn, B., and Muhlethaler, P.,"An aloha protocol for multihop mobile wireless networks," IEEE Trans. Inf. Theory, vol. 52,no. 2, pp. 421436, Feb. 2006

[14] Jayakody, D. N. K., "Novel Lossy Forwarding Scheme for Cooperative Wireless Networks," IET Electronics Letters, vol (8), no.25 pp. 2070 2072, Dec 2016.

[15] Nguyen, D. K., Jayakody, D. N. K., Chatzinotaz, S., Thompson, J., Li, J., "Wireless Energy Harvesting Assisted Two-Way Cognitive Relay Networks: Protocol Design and Performance Analysis," IEEE Access, DOI: 10.1109/ACCESS.2016.2644758, Dec 2016.

[16] Jayakody, D. N. K., Li, J., Mark, F. Flanagan,"A Soft Network-Coded Multilevel Forwarding Scheme for Multiple-Access Relay Systems," IEEE Transactions on Vehicular Technology, vol.65, pp.3430-3439, June 2015.

[17] Small Cell Forum release. 3GPP 3G femtocell standards overview. Version: 044.07.01, December 2013.

[18] V. Chandrasekhar, M. Kountouris, and J. G. Andrews, Uplink capacity and interference avoidance for two-tier femtocell networks, IEEE Trans. Wireless Commun., vol. 8, no. 7, pp. 34983509, July 2009.

[19] K. Huang, V. Lau, and Y. Chen, Spectrum sharing between cellular and mobile ad hoc networks: tranmission-capacity trade-off, IEEE J. Sel. Areas Commun., vol. 27, no. 7, Sept. 2009.

[20] L. K. Law, K. Pelechrinis, S. V. Krishnamurthy, and M. Faloutsos, Downlink capacity of hybrid cellular ad hoc networks, IEEE/ACM Trans. Netw., vol. 18, no. 1, pp. 243256, Feb. 2010 